\title{
Predictor packing in developing unprecedented shaped colloidal particles
}

\author{
Mubarak Ali ${ }^{a^{\prime *},}$ I-Nan Lin ${ }^{b}$ and C. $-J$. Yeh $^{\mathrm{c}}$ \\ a Department of Physics, COMSATS Institute of Information Technology, Islamabad \\ 45550, Pakistan, * Email: mubarak74@comsats.edu.pk, mubarak74@mail.com
}

${ }^{b}$ Department of Physics, Tamkang University, Tamsui Dist., New Taipei City 25137, Taiwan (R.O.C.). e-mail: inanlin@mail.tku.edu.tw

' Department of Engineering and system Science, National Tsing-Hua University, Hsinchu 30013, Taiwan (R.O.C.). e-mail: arayray220@gmail.com

\begin{abstract}
Developing particles of different geometric anisotropic shapes are the hot topic since decades as they guarantee some special features of properties not possible through other means. Again, controlling atoms to develop certain size and shape particle is a quite challenging job. In this study, gold particles of different shapes are developed via pulse-based electronphoton-solution interface process. Here, it is discussed that gold atoms under certain transition state amalgamated at solution surface to develop monolayer assembly around the light glow of electrons and photons, which is known in plasma, generating through flowing argon gas copper capillary, which is known in cathode. The rate of uplifting gold atoms to solution surface is controlled under the fixed optimized entrance of forced energy electron streams and photons of high forcing energy. Uplifting gold atoms dissociated on dissociation of precursor under the dissipating heat energy resulted by propagating photonic current through graphite rod immersed in the solution, which is known in anode. On the other hand, packets of nano shape energy resulted by the controlled tuned pulse protocol developing tiny particles of own shape by binding transition state atoms of compact monolayer assembly. At solution surface, adjusting atoms of monolayer tiny particle into onedimensional arrays under the disconcerted lateral forces following by their elongation
\end{abstract}


under uniformly exerted opposite poles forces. This results into convert them in a structure of smooth elements where adjacently placed electrons and those in the outer rings of elongated atoms inter-connecting side-to-side by introducing orientationalbased stretching of clamped energy knots. Tiny sized particles developed their atoms of one-dimensional arrays in structure of smooth elements exert an immersing force at favorable side tips and where many such tiny particles around the light glow work as one unit for each case resulting into pack by inter-connecting at inside their common point to nucleate the shape of certain particle. Depending on the development zone of such tiny sized particles and their amount of simultaneous packing under naturally maintained orientations develop their different geometric anisotropic shaped particles. At fixed precursor concentration, increasing the process time results into develop particles of low aspect ratio. Under tuned parameters, developing mechanisms of particles of high aspect ratio exhibiting unprecedented features are discussed.

Keywords: Fundamental and applied physics; Process parameters; Force-energy; Anisotropic particles; One-dimensional particles; Three-dimensional particles

\section{Introduction}

To design different sort of material, it is remained crucial since the birth of materials science. Intellectuals' and scientific leaders' fields of materials and interdisciplinary areas remained engaged to design specific categories of materials in all ages as per need and demand of on-going era. Among the categories of materials, the synthesis of materials in particles shape and size remained dominant in the past few decades as well as in the current ages and they hold a promising future too. To describe the scope and significance of tiny particles, nanoparticles, and particles, it is easy to say that several scientific leaders as well as emerging scientific leaders are working to reach out the required size and shape of particles in diversified class of materials along with their anisotropy and aspect ratio as such materials may be directly (indirectly) the origin of certain cutting-edge application that may not only bring scientific and technological impact but also social impact. In this context, various scientific journals covering all class of materials are publishing syntheses of particles under different means along with different targeted aspects of their applications. The explanations which are put forth on 
the development mechanisms of tiny particles, nanoparticles and particles are spanned over different class of opinions and scientific arguments.

But, undoubtedly, when the nanoparticles and particles showing the same morphology-structure (features of shapes) under their microscopic images, they raise a combine question i.e., "Why they contain a lot of diversified ranges of explanation and viewpoints". Obviously, it is to be; the scientific explanations vary process to process and within the same process also. The particles of same shape and structure are almost delivering similar sort of result along with their practical demonstration at forefronts of cutting-edge applications. In the synthesis process of nanoparticles and particles, facilitator of their atoms can be varied, but their electronic structure under unchanged dynamics is to be remained the same because of the same nature since existence.

Overall, to design material at nanoscale for getting unique benefits has always been the prime objective of scientific community and investigating structure under the least wavelength available at light-spectrum has great challenges. To manipulate and probe matter at nanoscale for development of new tools is critical for nanoscience and nanotechnology [1]. One-dimensional nanowires [2] and two-dimensional free-floating sheets have been developed by strong dipole-dipole interaction [3]. To structure matter on the scale of length comparable or smaller to the wavelength of light can deliver phenomenal optical properties [4,5] and catalytic activity of metallic nanostructures, enhanced significantly on controlling phase $[6,7]$.

Formation of tiny clusters and their coalescence into extended shape of particles is one of the long and continuously over-looked phenomena [8-18]. Nanometer-sized gold clusters behave like simple chemical compounds and may find a wide range of applications in catalysis, sensors and molecular electronics [8]. The discrete nature and stability of nanocrystals along with their tendency to form extended supperlattices suggest ways and means for the design and fabrication of advanced materials having controlled characteristics [9]. Development of single crystal by the aggregation of nanocrystals appears as a realistic goal [10]. The development of new, ultimately small, electronic devices is one of the most prominent and potentially long-term applications of nanoparticles technology [11]. Self-assembly offers a very promising route to construct complex shapes at nano-/micro-meters level and engages many of the classical 
disciplines of science and engineering [12]. A grand challenge is to assemble and position the nanoparticles at preferred sites which will enable the construction of complex and higher-order functional structures [13]. To organize nanometer-sized building blocks into specific shapes is one of the current challenges [14]. On successful assembling of colloidal particles into useful structures the 'atoms' and 'molecules' will become tomorrow's materials [15]. Understanding the electronic absorption and dynamics of individual nanoparticles is pre-requisite for their development into an ordered array instead of their agglomeration [16]. Possibility to coalesce nanocrystals allows one to develop materials with abundant selections, which leads to the opening of entirely new field [17]. On controlling precisely, the surface properties of nanoparticles will lead to direct their assemblies into higher order structures [18]. Tiny particles are molecular-like structures and certain numbers of atoms form hcp structure [19]. Shape and size of metallic structures have remained under the extensive debate and generated significant interest in several newly emerging areas of nanoscience and nanotechnology [20-22]. It has been shown that shape entropy drives the phase behavior of systems of anisotropic shapes through directional entropic forces [23]. Geometry and entropy of colloidal particles not only explain the structure but dynamics also [24]. In a known protocol, in addition to the disordered jammed configuration, there are ordered metrics capable to characterize the order of packing [25]. Those studies are the growth and beauty of science behind materials of technological advance.

Efforts have also been made to synthesize tiny clusters and anisotropic shapes of gold by employing various plasma solution methodologies. Mainly, four approaches remained under operation: (1) DC plasma discharge in contact to liquid [26-29], (2) DC glow discharge plasma in contact to liquid [30], (3) pulse plasma discharge inside the liquid [31-36], and (4) gas-liquid interface discharge [37]. Hydrogen peroxide was the most probable reaction mechanism to synthesize gold nanoparticles [26-29]. While synthesizing spherical-shaped nanoparticles, electron flux at a given current remains constant on the surface of solution [32] and plasma electrons act as cathode [33]. Hydrogen radicals generated in the discharge where their rate increased consistently with the external field and due to dissolution of nanoparticles, reduction rate lowered and was the cause of anisotropic growth [34]. The influence of Brownian motion along 
with the surface charge of nanoparticles explained their stability [35] and negatively charged surface of nanoparticles kept them away from agglomeration [36]. Gold nanoplates and nano-rods developed at the surface of solution and spherical-shaped particles in the solution [37].

The fundamental process of formation of various tiny-sized particles under different process conditions is discussed elsewhere [38]. The development of gold nanoparticles and particles under varying precursor concentration has been discussed elsewhere [39]. Tapping opportunity of tiny shaped particles and role of precursor in developing shaped particles under the same setup are given elsewhere [40]. Different shaped tiny particles along with their extended shapes developed under varying the ratio of bipolar pulse and different pulse polarity [41]. Development of extended shaped particles at increasing process duration along with the adjacent and lateral placement of electrons in atoms of tiny particles while developing structure of smooth elements are discussed elsewhere [42]. A detailed study presented about the development mechanism of tiny shaped particles dealing localized gravity of their mono layer at solution surface while developing its certain shape particle where one-dimensional arrays of atoms converted into structure of smooth elements [43]. Atoms of electronic transitions elongate or deform but don't ionize where forcing energy propagating through inter-state electron gap is related to photonic current instead of electronic current [44]. Atoms of electronic transitions but executing confined inter-state electron-dynamics under the involvement of conservative forces where engaging the conservative energy are discussed while evolving their different dimension and format structure [45]. The phenomena of heat and photon energy are discussed elsewhere [46] where silicon atom was taken as a model system. Atomic behaviors Along with field force behaviors are quite diligent in showing the performance of nanoparticles while using them as nanomedicine [47]. Recently, study on switching dynamics of morphology-structure in carbon deposited films is reported elsewhere [48] where a slight variation in the process conditions resulting into alters morphology-structure of evolving grains and crystallites.

To synthesize tiny sized particles and large sized particles of different possible materials, it is an easy task, but it is difficult to understand the underpinning mechanism of their development. The present work describes the fundamental process of formation 
of triangular-shaped tiny particles at solution surface in relation to predictor packing and developing particles of different geometric anisotropic shapes under the exerting forces while employing the pulse-based electronphoton-solution interface process. Under set parameters, gold atoms dissociated from the precursor as per absorbing energy transferred through immersed graphite rod, they uplifted to solution surface against the reaction of entrance of forced energy electron streams and high energy photons where packets of nano shape energy generated by tuned pulse protocol bind atoms of monolayer assembly into their own shape working for predictor packing at solution surface to develop particles of unprecedented shapes.

\section{Experimental details}

Hydrogen tetrachloroaurate (III) trihydrate was purchased from Alfa Aesar and different concentrations of solution were prepared after mixing with DI water. A layout of pulsebased electronphoton-solution interface process was designed in the case of present experiments is shown in Figure 1. Copper tube internal diameter $3 \mathrm{~mm}$ along with flowing argon gas was utilized as a source of forced energy electrons streams and forcing energy photons where $100 \mathrm{sccm}$ flow rate of argon gas was maintained through digital mass flow controller. Graphite rod (width: $1 \mathrm{~cm}$ ) was immersed in the solution which is also known as anode. The bottom of the copper capillary was adjusted just above the surface of solution $(\sim 0.4 \mathrm{~mm})$ and distance from the graphite rod was kept 4 $\mathrm{cm}$. Symmetric-bipolar modes of pulses were employed which were generated by the pulse DC power controller (SPIK2000A-20, MELEC GmbH Germany). Temperature of the solution was recorded from the distance of one meter by LASER-guided meter (CENTER, 350 Series); $21^{\circ} \mathrm{C}$ at the start and $47^{\circ} \mathrm{C}$ at the end of 20 minutes with $\pm 1^{\circ} \mathrm{C}$ accuracy. The precursor concentration was $0.3 \mathrm{mM}$. Total quantity of solution was 100 $\mathrm{ml}$. The processing time of the solution was 20 minutes. In each experiment, the average input DC power was measured to be 36 (watts) where running voltage was $\sim 30$ (volts) and current was $\sim 1.2$ (amp) and light glow sustained after few seconds under the slight variation of input power; power fluctuation was more at the start of the process (initial few seconds), which has almost stabilized in the duration of the afterward 
process. Step-up transformer increased voltage 40 times. Bipolar pulse ON/OFF duration in each experiment was set $10 \mu \mathrm{sec}$.

Thickness of the copper capillary wall was $1.5 \mathrm{~mm}$, whereas, the diameter of inner hollow space through which argon gas flows was $3 \mathrm{~mm}$ as shown in Figure S1. Zones related to placing packets of nano shape energy under controlled tuned pulse protocol, impinging electron streams and traveling photons of different wavelengths at the surface of monolayer gold atoms are pointed out by (7), (8) and (9), respectively in Figure 1. Further details of these are given in Figure S2. To characterize features of various particles both bright field and high-resolution microscope images of particles were captured (TEM, JEOL JEM2100F; operated at $200 \mathrm{kV}$ ). Prior to those investigations, a drop of solution from each prepared concentration was poured on copper grid and was kept in Photoplate degasser (JEOL EM-DSC30) for the elimination of moisture.

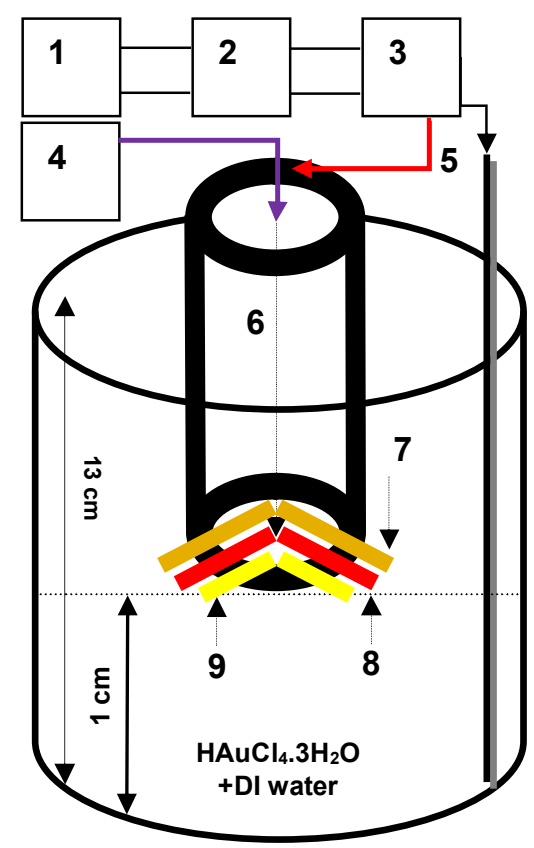

Figure 1: Layout of pulse-based electronphoton-solution interface process; (1) power supply, (2) pulse power controller, (3) step-up transformer, (4) argon gas, (5) graphite rod, (6) inner (hollow) region of copper capillary, (7) region of placing nano shape energy (outer region of light glow), (8) electron-solution interface (middle region of light glow) and (9) photon-solution interface (inner region of light glow)

\section{Results and discussion}

Surplus photonic current under set power propagates through the graphite rod, which is 
working for the positive terminal as known conventionally in the flow of current through wire while it is known as anode in the case of electrochemistry. Mainly, the photonic current propagates through the wire known in negative terminal which is connected to copper capillary dealing flow of argon gas also. The flowing argon gas splitted into the electron streams at the bottom of copper capillary. Because of none availability of unfilled states in the argon gas atoms and due to excess given field of propagating photons characteristic current through their inter-state electron gap resulting into split them where light glow is observed, which is known in plasma and that glow is just at the bottom of the copper capillary, which is known in cathode. However, resulted heat energy dissociating gold atoms from the precursor is negligible. Gold atoms mainly dissociated from the precursor by energy provided by the photons characteristic current in the form of heat energy. That heat energy is resulted from the interacted photons characteristic current to solution as they propagated through inter-state electron gap of graphitic state atoms of the immersed graphite rod. The uplifting of metallic atoms to solution surface is under the reaction of entering forced energy of electron streams and forcing energy of high energy photons. The high energy photons entering in the solution are resulted because of the splitting flowing inert gas atoms as they didn't work for forced energy of splitted electron streams.

It is discussed elsewhere [38] that both electron streams and photons result on splitting of flowing inert gas atoms. Photons of high energy travelling more in normal to their points of generation entered in the solution instead of traveling along the air-matter interface [41]. Argon gas was used to activate the process of developing gold particles. Splitting of inert gas atoms not only delivered electrons to impinge underlying gold atoms but also enabled the propagation of photons characteristic current to inter-state electron gap prior to split resulting into allow them to travel in increasing wavelength in the open medium. Therefore, a certain amount of flowing inert gas atoms split just over the solution surface where photons increasing in their wavelengths work at a place utilizing forcing energy to align underlying electronic structure of elongated triangularshaped tiny particle in the case of perturbed electron states of atoms, thus, modify further the structure of elongated atoms of tiny particle into structure of flatten smooth elements as discussed elsewhere [43]. The inert gas atoms split under the application 
of excess propagation of photons characteristic current through their inter-state electron gaps for which further detail is given elsewhere [44].

Auto-controlled current for set pulse duration propagating through copper tube gave packets of nano shape energy under the application of step-up transformer where tuned pulse protocol was controlled during each pulse ON/OFF time cycle. Underneath copper capillary where monolayer assembly of gold atoms is developed, packets of nano shape energy are placed horizontally over them resulting into bind atoms of monolayer assembly into tiny particles of own shape nearly all around the region of light glow at solution surface. The round surface width i.e. $1.5 \mathrm{~mm}$, which is approximately equal to the thickness of wall of copper capillary, is shown in Figure S1. Therefore, packets of nano energy shape-like connected triangles developed tiny sized particles of monolayer in their own shape. The gold atoms when bound into monolayer tiny sized particle at solution surface under the energy of triangular-shaped packet they were under the recrystallization state as discussed elsewhere [43]. The placed horizontally doublepackets of nano shape energy over the surface of solution bound gold atoms of transition state where the placement of electrons was more along their poles under the exertion of naturally available surface format force. As graphite rod is immersed into the solution from bottom level to top level surface, surplus propagation of current occurs into it, on leaving the propagation of inter-state electron gap, it enters in the solution to dissociate the gold atoms from the precursor. On the other hand, entering forcing energy of photons along with splitted electron streams uplifting those dissociated gold atoms under the force of reaction resulting into develop monolayer assembly of gold atoms. Due to the feature of bipolar pulse having unity ratio of pulse OFF to ON time, each tiny particle is first developed in shape-like two joint triangles. The details of their separation into two equal equilateral triangular-shaped tiny particles due to a bit perturbed axis are given elsewhere [39-43].

Figure 2 shows dissociation of gold atoms under the supply of heat energy through immersed graphite rod and their uplifting to solution surface under the entering forced energy splitted electron streams and forcing energy photon in high energy. These both constitute the light glow (plasma), thus, hereafter, where a light glow is referred it is related to both forced energy electron streams and forcing energy photons. 


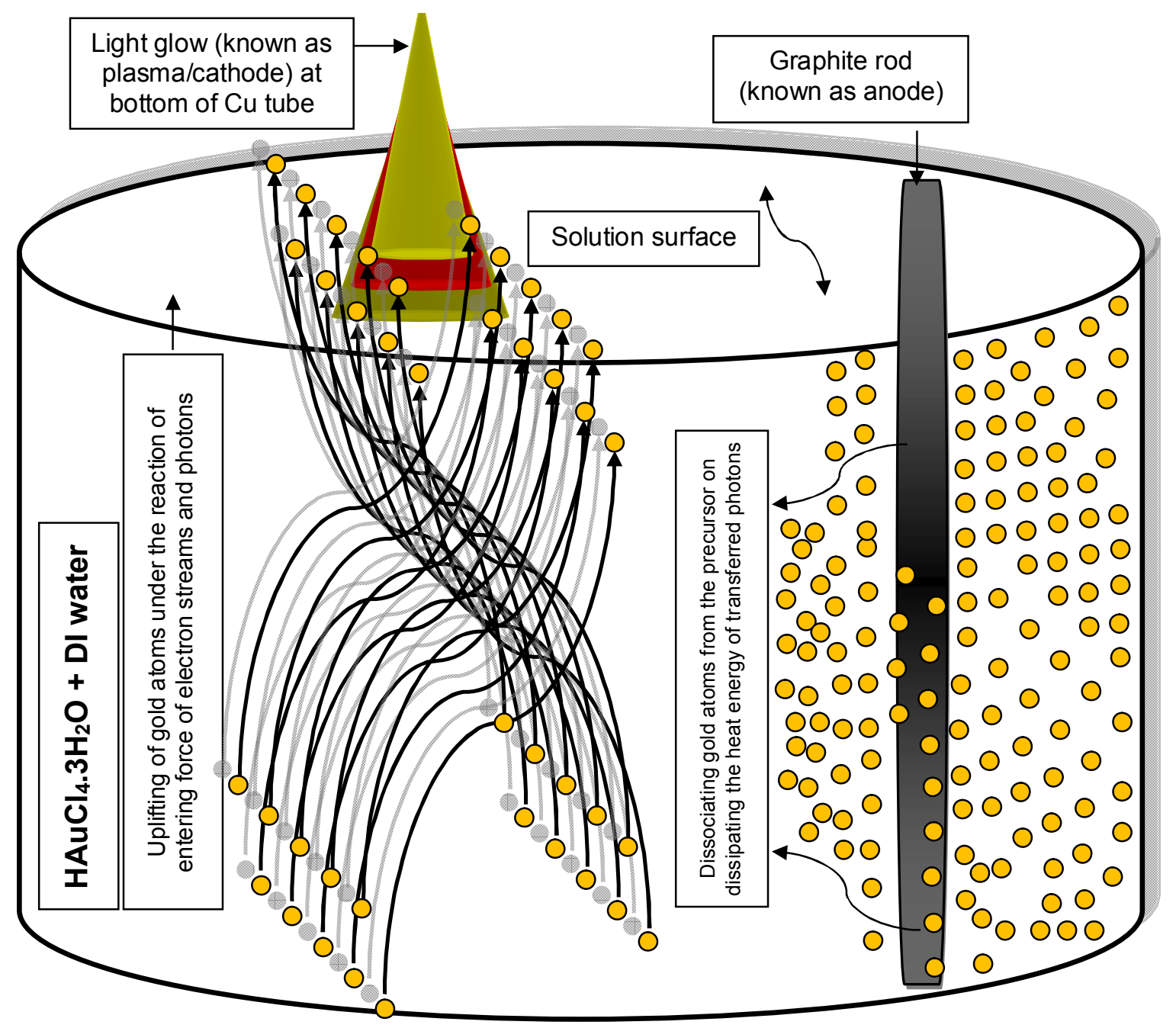

Figure 2: Gold atoms dissociating from the precursor and uplifting to solution surface

Signatures of nano shape energy, electron streams and light glow dealing 3D space while interacting circular solution surface are shown in Figure S2. Therefore, interactions of nano shape energy, electron streams and photons are consecutively (one at one time) in three cones like shapes; outer cone shape is belonged to the placing packets of nano shape energy, middle impinging electron streams and inner one is belonged to photons travelling along the interface and entering in the solution, also. High energy photons enter in the solution under the strong impact while low energy photons dissipate heat energy just over the surface of solution where their poor interaction takes place.

At solution surface, gold atoms under transition state construct monolayer assembly nearly in the circular form but by exempting the regions of zero-force axis as shown in 
Figure 3. Placed nano shape energy bound transition state atoms of monolayer assembly into tiny sized particle of nearly in own shape where having the monolayer but three-dimensional structure of surface format but that is known in hcp structure or twodimensional structure. A detail study on structural evolution in different formats in certain solid atoms of electronic transition is given elsewhere [45] where the distribution of surface format forces for different poles is also shown. During processing $\mathrm{AgNO}_{3}$ solution, there was no formation of triangular-shaped tiny particles even though same process conditions were employed indicating that silver atoms deal transition state (recrystallization state) to elongate is at different level of ground surface instead of the gold atoms as they are elongating at solution surface set for the same height as for the case of processing silver solution. In the case of gold solution, it was not like silver and entire solution in the beaker changed into a uniformly changing color rapidly. In the case of gold solution, color of solution was changing at fast rate in the initial few seconds due to utilization of atoms to bind for their tiny sized particles, nanoparticles and particles.

Placed double-packets of nano shape energy bind atoms of monolayer assembly into two connecting triangular-shaped tiny particles, in each case, binding them in their own shape are shown in Figure 3; zones related to photons at work (and light glow), impinging electron streams and placing horizontally packets of nano shape energy are in the central region, in the middle region and in the outer region, respectively, as roughly sketched in the figure. At centre of light glow, a developing mono layer of certain shape particle is also shown along with several earlier developed mono layers as indicated by the arrow signs where sinking one step down while dealing the localized gravity. Binding of such mono layers in developing particle of certain shape is through the lateral placement of electrons in their partially elongated atoms. Further detail of binding mono layers in developing certain shape particle while dealing the localized gravity at solution surface is discussed elsewhere [43]. Such tiny sized particles work and directed as one unit in each case from the regions of formation to pack at already allocated unfilled regions to develop certain shape particle under the force as per their rate of development in different zones of solution surface. Structures of smooth elements of triangular-shaped tiny particles in the reach of packing at centre of light glow further elongate while impinging electron streams at fixed angle. Structures of 
smooth elements further shape and acquire smoothness under the forcing energy of high density travelling photons at the instant of (on) packing.

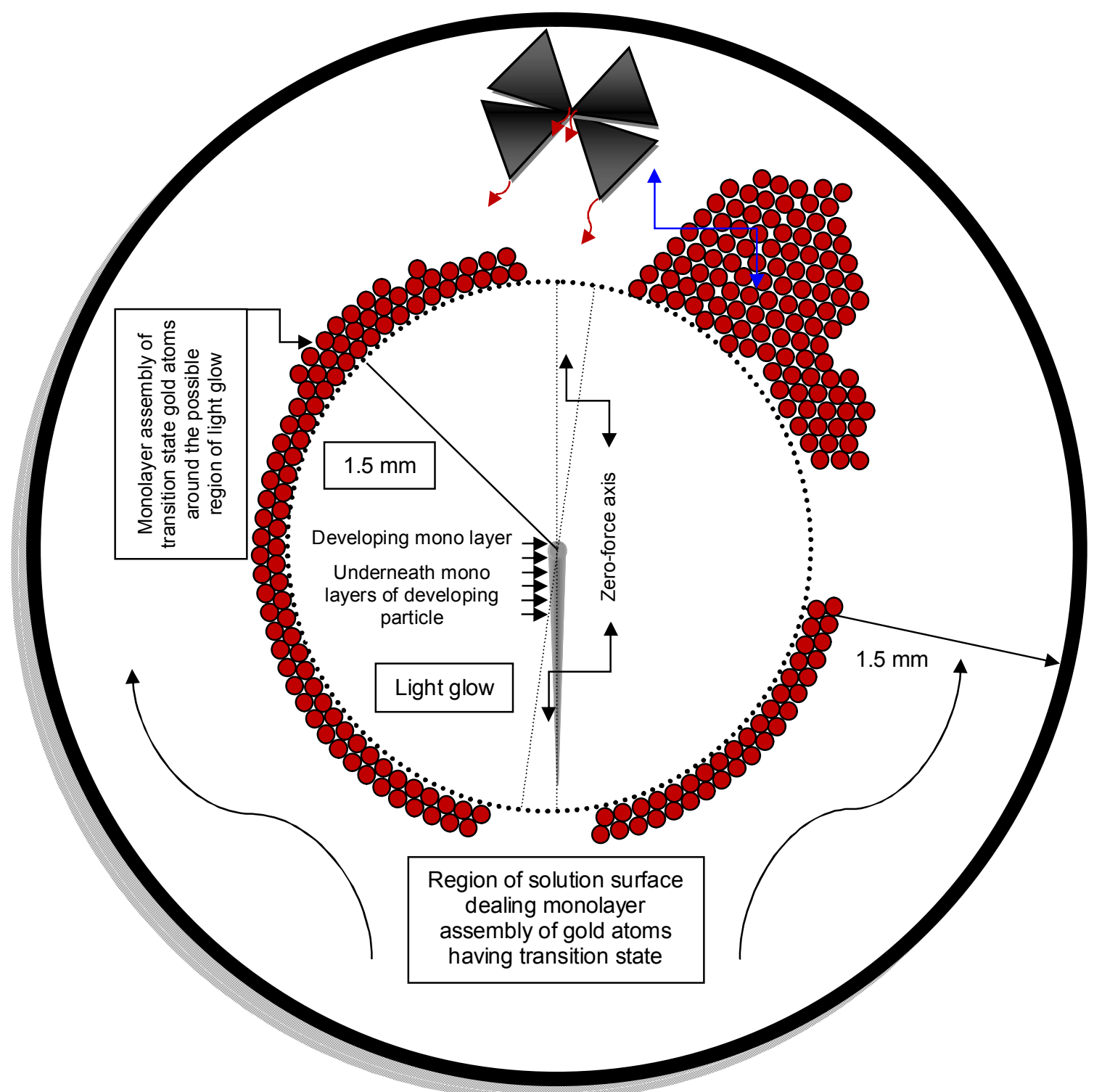

Figure 3: Gold atoms at solution surface in suitable circular zone width i.e. $1.5 \mathrm{~mm}$ where doublepackets of nano shape energy placed over monolayer assembly to bind transition state atoms in own shape and size where no amalgamation of the atoms along the zero-force axis occurs

The predictor packing of triangular-shaped tiny particles is from the same region where they were developed. At tuned bipolar pulse mode (pulse ON/OFF time: $10 \mu \mathrm{sec}$ ) along with other set parameters as described in the experimental details, the each developed tiny sized particle contained the two connecting triangular-shaped tiny 
particles. Two joint triangular-shaped tiny particles separated into two equal size triangular-shaped tiny particles under the difference of force influencing at opposite pole between their connected atoms. However, triangular-shaped tiny particles developed directly under the application of unipolar pule as discussed elsewhere [41]. It can be observed in the illustration given in Figure 3 where tiny sized particle of two connecting triangles developed under the application of double-packet nano shape energy.

The left one (companion) tiny particle is in queue to follow the packing of earlier one in each case is indicated in Figure 3; the left one triangular-shaped tiny particle turned and directed tip towards the region of packing dealing the force of surface format, a bit in tilted manner, till reaching at centre of light glow. In the similar manner, elongated triangular-shaped tiny particles are arriving to pack at pre-allocated regions of developing monolayers of developing certain shape particle where the packing at any point results into the sinking of particle on termination under the force of gravity where each layer binds under the mechanism of lateral placement of electrons as discussed elsewhere [43]. In the case of rod-/bar-shaped particles, tiny particles were developed at oppositely-sided regions available at the sides of rear north-south axes as illustrated in Figure S3. Such shaped particles are said to be particles of one-dimensional shape but binding (packing) of tiny particles in developing their structure remained two-sided (opposite to each other from the centre point) and they possess more width of their developed structure of smooth element as compared to particles of three-dimensional shapes where binding (packing) of tiny particles in developing their structure remained three-sided.

In the case of plate-like particles, monolayer assembly cropped into triangularshaped tiny particles as well due to the availability of atoms at solution surface along east west poles around pulse-based electronphoton-solution interface. To develop triangular-shaped particle, at one time, the momentum of packing remains the same from all three sides where each tiny particle packing secures orientation at $120^{\circ}$ angle and from three different zones under the force toward their respective regions of packing. To develop hexagonal-shaped particle, at one time, the momentum of packing remains the same from all six sides where each tiny particle packing secures orientation 
at $60^{\circ}$ angle and from six different zones under the force toward their respective regions of packing and so on.

Figure 4 shows formation of connecting triangular-shaped tiny particles where the size is decreasing under the prolonged process duration. As discussed above, such tiny particles separated into equal triangular-shaped tiny particles while exerting the force of opposite pole between the points of their joint atoms. Decreasing the size of triangularshaped tiny particles resulted into decrease the aspect ratio of their particles as they are being developed in the presence of reduced amount of gold atoms and at later stage of the process. At start of the process, mainly large-sized tiny particles were developed and their packing into particles possesses large aspect ratio too. As the size of the tiny particle decreases on reducing the number of atoms per unit area at solution surface, particles of decreasing aspect ratio develop with time even though they possess the same shapes as for those developed at initial stage of the process. Under the fixed concentration of precursor, the number of amalgamating atoms into monolayer assembly reduced significantly resulting into lower the aspect ratio of particles developed with the passage of prolonging the process time. It is estimated that in the beginning of the process, the size of tiny particle was varied depending on the ratio of pulse OFF to ON time.
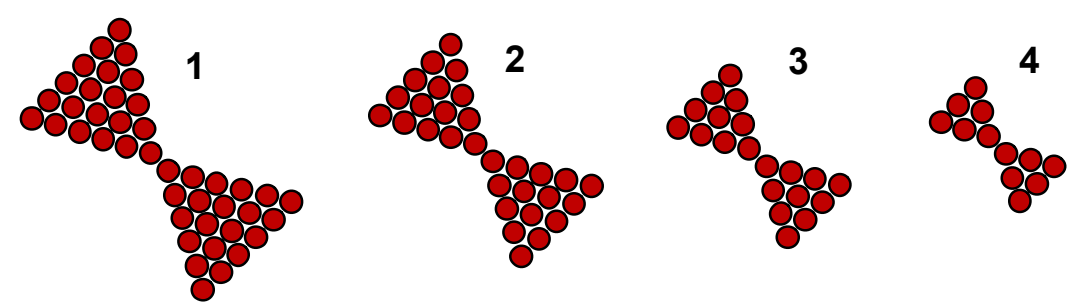

Figure 4: Connected triangular-shaped tiny particles decreasing size with respect to process time while dealing the fixed precursor concentration of the process

Each developing layer of the geometric anisotropic shaped particle self-deal the packing as vacant left place which provides the force to triangular-shaped tiny particle in the relevant zone of developing particle where those places pack for fit size at exact time and fill that vacant allocated place, therefore, it is also subjected to self-filling of unpacked regions besides the predictor packing as referred. A complete layer of developing geometric anisotropic shaped particle developed in each step of pulse ON 
time $(10 \mu \mathrm{sec})$ where they possess structure of smooth elements. That developed monolayer structure of smooth elements went down to solution surface while dealing the localized gravity and followed by developing new layer starting exactly at the centre of the one prior developed, in the same size and shape, as the formation of triangularshaped tiny particles along with predictor packing remained the same for the period of formation of that particle. While developing upper layer, downward layer descends level upto $\sim 0.3 \mathrm{~nm}$ (an approx. diameter of gold atom), however, under the break of developing layers for next set period of pulse ON time (10 $\mu \mathrm{sec})$, the particle sinked by leaving the solution surface completely resulting into leave the centre of light glow at solution surface, thus, allowing to develop a new particle. Once, the process of formation of new layer in developing certain particle is timed out; the particle is no more under growth. This leads into start the developing process of the new particle. On utilizing the developed tiny particles around centre, the newly developed tiny particles started utilizing their predictor packings, thus, developing new particles of geometric anisotropic shapes. Lower the number of binding mono layers, then, higher is the aspect ratio of resulted particle. The particle's shape varies depending on the region of initially packed triangular-shaped tiny particles, at one time, along with their quantity; when three equal size tiny shaped particles packed simultaneously at $120^{\circ}$ angle placed side by side, it will result into develop triangular-shaped particle and when six equal size tiny shaped particles simultaneously packed at $60^{\circ}$ angle placed side by side, it will result into develop hexagonal-shape particle.

Figure 5 shows particles of various anisotropic shapes which developed under predictor packing of triangular-shaped tiny particles. The differently shape shown particles in Figure 5 clearly figure out the degree of orientation at which predictor packing takes place and in each face of the particle by taking the centre of particle as a reference point. Therefore, photons worked at the place help in constructing perfect smooth elements having their equal inter-spacing distance in each forming layer of developing particle as illustrated in Figure S4. 


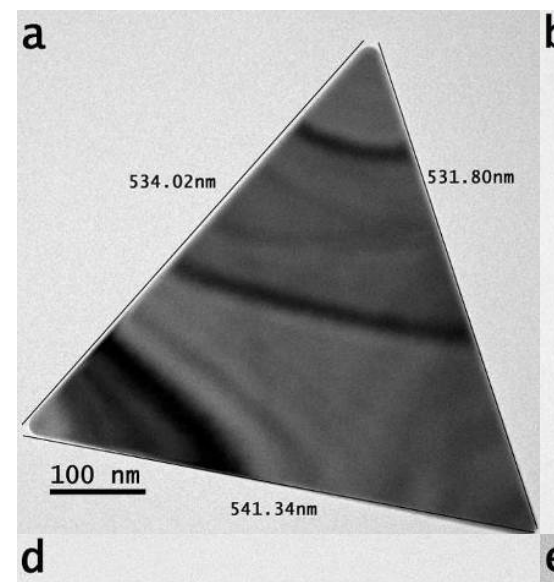

b
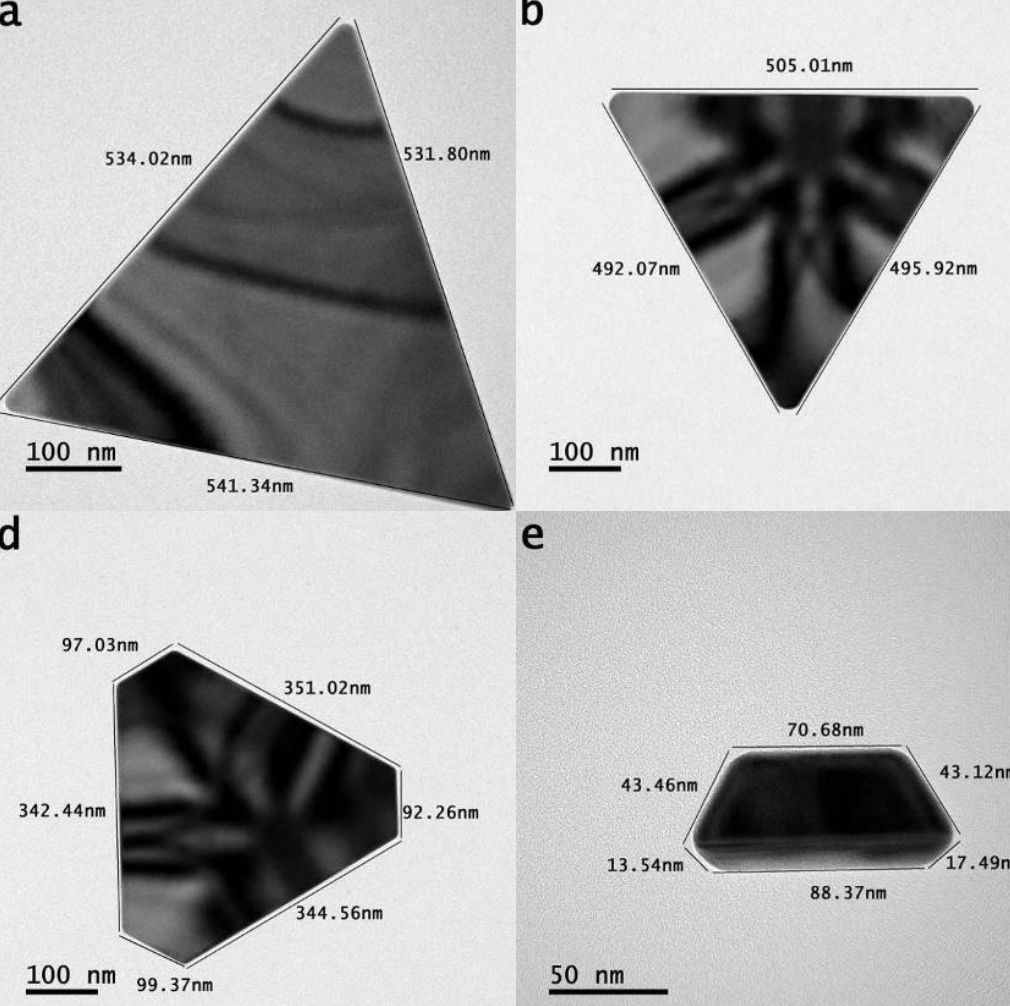

g

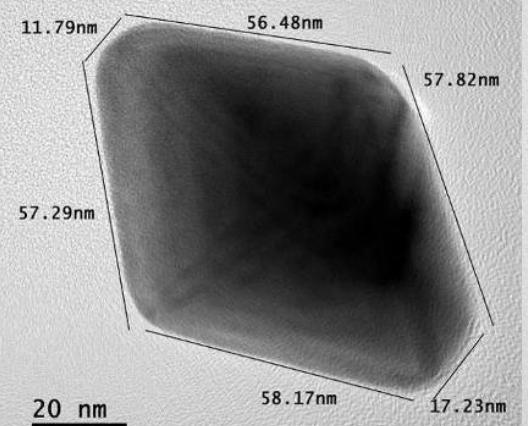

j

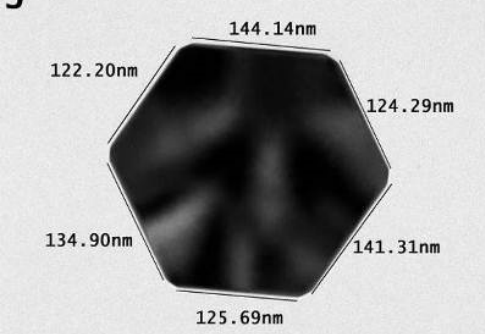

$100 \mathrm{~nm}$ e

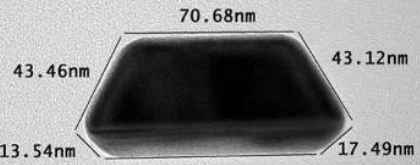

$88.37 \mathrm{~nm}$

\section{$50 \mathrm{~nm}$}

h

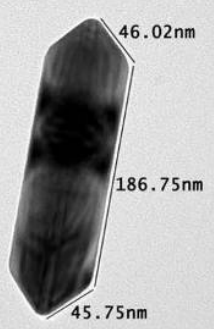

$100 \mathrm{~nm}$

k

$100 \mathrm{~nm}$

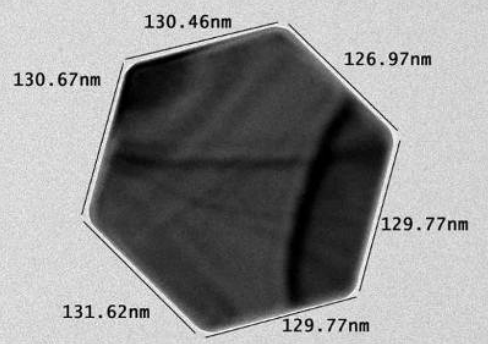

C

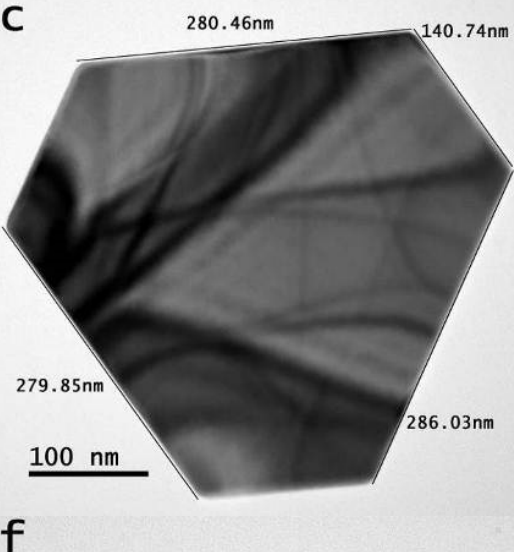

f

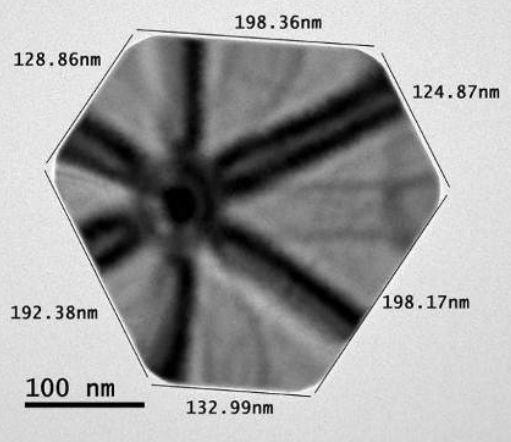

i

$24.92 \mathrm{~nm}$

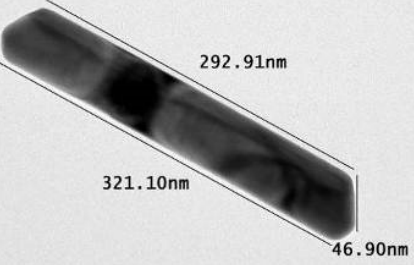

$100 \mathrm{~nm}$

1

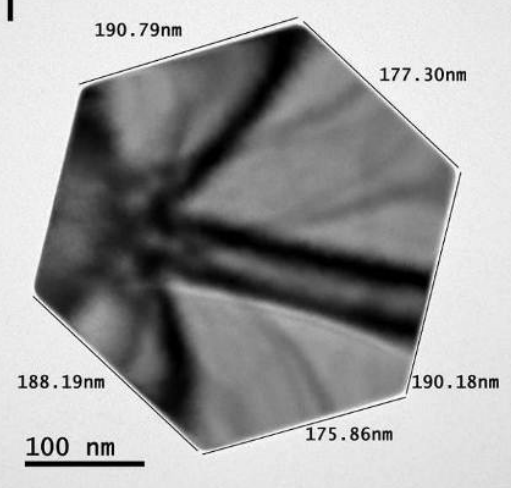


m

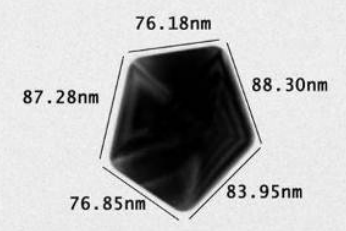

$100 \mathrm{~nm}$ n

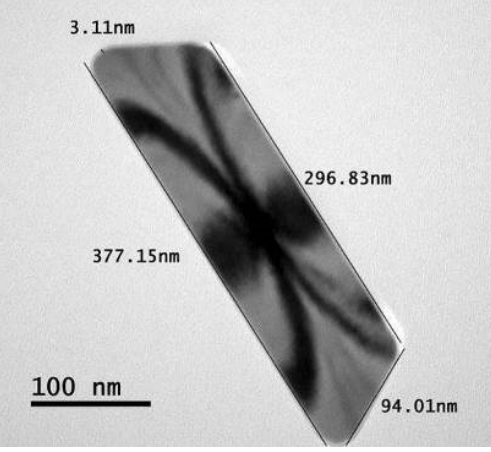

0

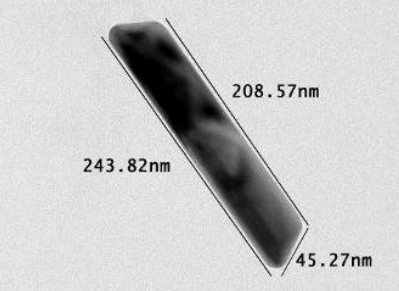

$100 \mathrm{~nm}$

Figure 5: (a-o) bright field transmission microscope images of standalone triangle-, hexagon-, isosceles trapezoid-, rhombus-, rod-, pentagon-, tape- and skating-like particles

In addition to several geometric anisotropic shaped particles shown in Figure 5, particles of various geometric anisotropic shapes together with distorted ones developed as well, which are shown in Figure 6 where they possess different size and shape. In those particles, some of them possess very small size while others possess very large size despite of that they have the same shape in some cases.

a

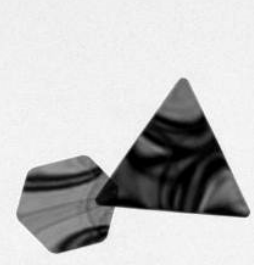

$200 \mathrm{~nm}$

d

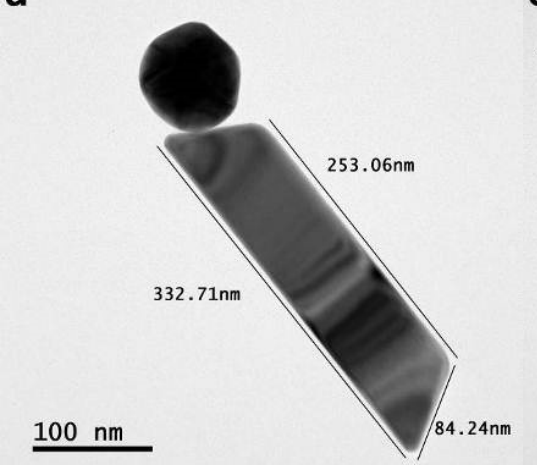

b

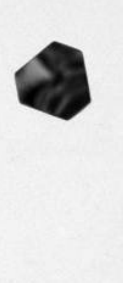

$100 \mathrm{~nm}$

e

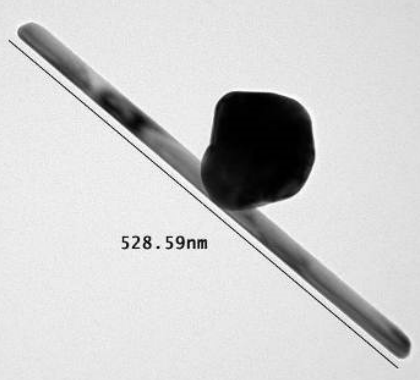

C

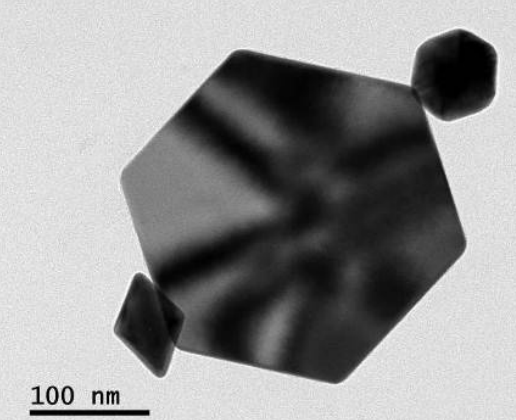

$100 \mathrm{~nm}$
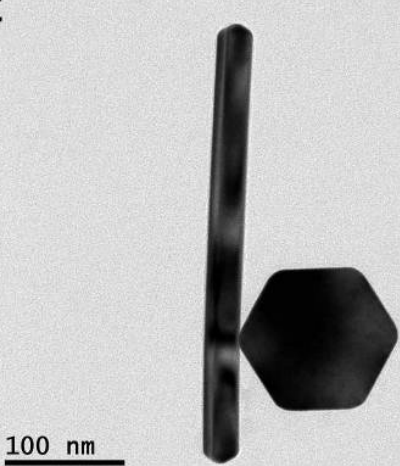

$f$

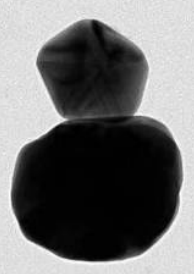

$100 \mathrm{~nm}$ 
g

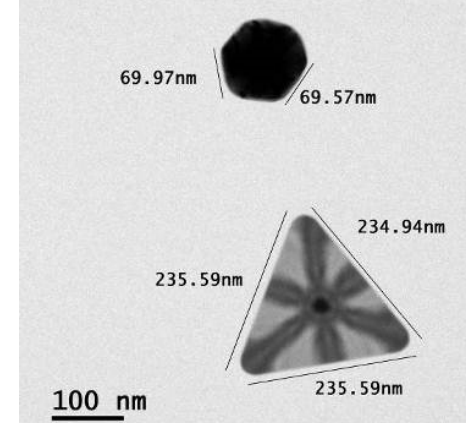

j

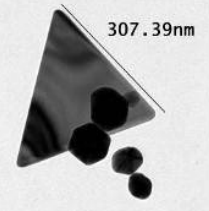

$200 \mathrm{~nm}$

m

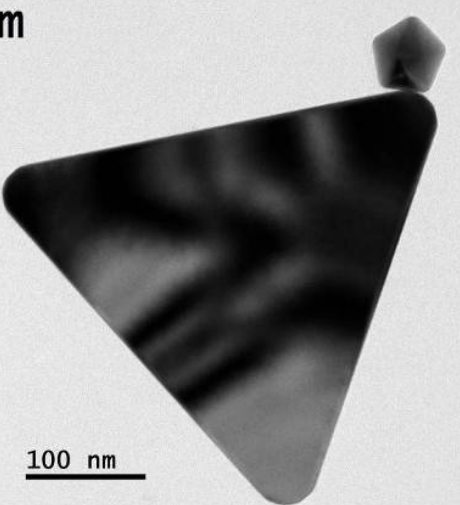

h

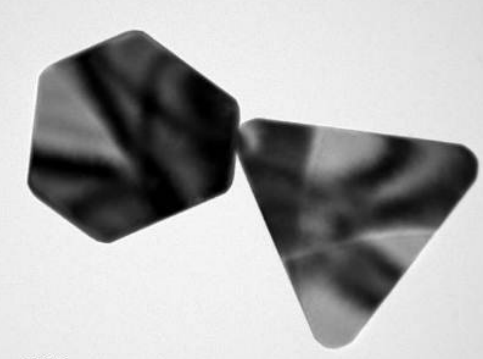

$100 \mathrm{~nm}$

k

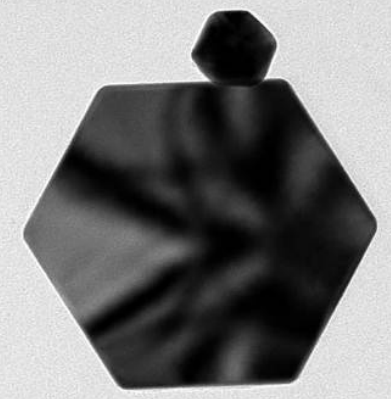

$100 \mathrm{~nm}$

n

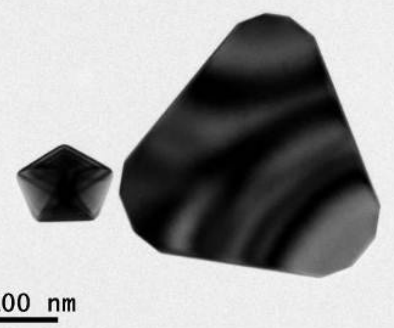

i

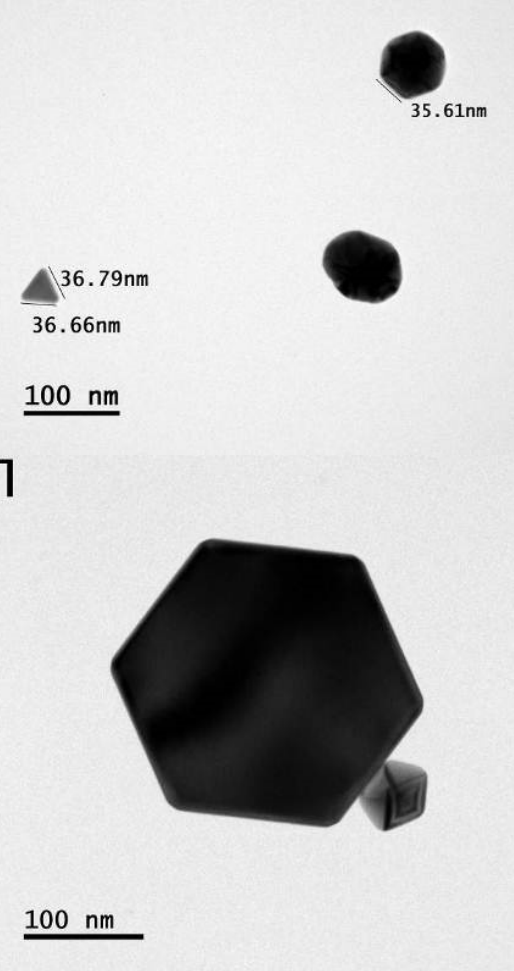

$100 \mathrm{~nm}$

Figure 6: (a-n) bright field transmission microscope images of various geometric anisotropic shaped particles; in some cases, they are coupling to each other, in some cases, they keep the distance, and, in some cases, they adhere to the distorted particles

Atoms of monolayer assembly at solution surface remained bound under the difference of force of oppositely faced poles. Once a gold atom (and atoms of other elements belonging to same family) comes at ground level (state), it exerts only single force, which is at dominating end. But prior to uplift gold atoms, they were at their solid state. So, just at the surface of solution triangular-shaped tiny particles, they exert localized force applicable in their regions for packing into particles of various shapes. 
On terminating the packing of developing large-sized particles, they sink under free fall. The developing of various geometric anisotropic shaped particles and their sinking enables arriving new stock of gold atoms at air-solution interface, thus, forming new monolayer assembly at air-solution interface. The binding of mono layer developed in certain shape of developing particle bind to another layer placing over it under the lateral placement of electrons in their atoms where expansion and contraction of their clamping energy knots contribute. Expansion and contraction in the case of atomic behavior when in transition for liquid state under varying orientating gravitational force and varying orientating levitational force for electrons is discussed elsewhere [50]. Atoms of one-dimensional arrays while in tiny particle or in large size particle maintain inter-spacing distance in addition to show only elongation of atoms. In the case of impinging electron streams from the external source, elongated atoms of tiny particles under opposite poles forces are further elongated. Thus, each tiny particle works as one unit while packing where new set of atoms arrive at solution surface under the reaction of force of entering splitted forced energy electron streams (of argon atoms) and photons of different wavelength just under the bottom copper tube. Forced energy (punched photons) are entered to solution through high density penetration of splitted electron streams of argon atoms along with photons of exceeding wavelengths generating normal to the solution surface. The photonic current propagated through elongated atoms of tiny grains of carbon film under the accelerated density resulting into the enhanced field emission as discussed elsewhere [49]. The origin of an atom to be in gas state or to be in the solid state along with structure of an atom is discussed elsewhere [50].

Photons propagated through graphite rod, they provided energy to dissociate gold atoms from the precursor at solution surface, thus, forming monolayer assembly around the pulse-based electronphoton-solution interface is under the reaction of forced energy provided by the electron streams along with the increasing wavelength of photons in the light glow developing centre of gravity at solution surface as well. Therefore, entering photons of different wavelengths along with the penetration of forced energy electron streams in the solution in the region forming the light glow don't appear to dissociate metallic atoms from precursor in such a large number and their systematic placement at 
solution surface while uplifting in each cycle of bipolar pulse ON time is under the reaction of that entering forced energy electron streams and forcing energy (photons) of high density. Therefore, uplifting of atoms to solution surface is because of the providing forcing energy through light glow while the dissociation of metallic atoms from their precursor is under the immersed graphite rod. Thus, developing monolayer assembly at solution surface under the uplifting of metallic atoms to solution surface is not only because of the one component but involving both light glow known in plasma generating at point of copper bottom known in cathode and propagating photons characteristic current through graphite rod known in anode also.

\section{Conclusions}

Different high aspect ratio shapes of geometric gold particles develop in pulse-based electronphoton-solution interface process under optimized process parameters. Under the controlled excess input power, high density of photons characteristic current propagated through immersed graphite rod transformed into heat energy on entering solution resulting into dissociates gold atoms from the precursor. When force behind electron streams enters in the solution, as a reaction, dissociated gold atoms are uplifted. Under controlled tuned pulse protocol, flowing argon gas atoms splitted into electron streams. Forced energy (punched photons) enable impinging of electron streams to underneath tiny sized particles accelerating to pack under immersing force exerting at tip of each their structure of smooth element. When not impinging in the absence of underneath matter at solution surface, forced energy electron streams enter to solution. In addition of entrance of forced energy electron streams in the solution, high energy photons in high density also enter in the solution. They enable the uplifting of dissociated gold atoms under the reaction of entrance generating at the bottom of copper capillary under the tuned pulse protocol.

Monolayer assembly developed around the light glow renovated in each pulse OFF cycle because of uplifting the gold atoms in each pulse ON time. The placing packets of nano shape energy, horizontally, under controlled tuned bipolar pulse protocol, they result into develop joint triangular-shaped tiny particles of monolayer following by the separation into two equal equilateral triangle shape tiny particles. Atoms of each tiny 
particle of triangular shape elongate under the force of surface format working along opposite poles from their centers where each one-dimensional array of atoms converts into the structure of smooth element, thus, working as one unit to pack at centre of light glow under the immersing force because of the design of process. A tiny particle doesn't go for pre-allocated region of packing of developing particle unless it has geometry of equilateral triangle shape. Triangular-shaped tiny particles structure of smooth elements initiate packing at pre-allocated regions first at the centre of light glow following by unfilled regions of developing mono layers in developing different shape particles of high anisotropy. The developed feature of tiny particles self-ensured the points (regions) of their packings, thus, their packing results into develop certain shape particle which possesses unprecedented features not possible to attain through other means.

On separation into two equal triangular-shaped tiny particles, a tiny particle turned and directed towards already reserved region of developing particle and same is the case for the companion tiny particle, but packs aside to the earlier packed one. Prior to assemble as one unit a tiny sized particle developed in structure of smooth elements, they elongated further while impinging forced energy electron streams at electronsolution interface. The numbers of initially packed tiny particles, at one time, from different zones of solution surface nucleate a particle of certain shape where initial number of simultaneously assembling tiny particles flowing by continuity in the packing for each developing mono layer of developing particle determine the shape and aspect ratio of particle. The synchronization of the setup resulted into develop several unprecedented featured particles in different sizes and shapes. Present study sets new trends in the field of materials science, physics, nanoscience and nanotechnology.

\section{Acknowledgements}

Mubarak Ali thanks National Science Council (now MOST) Taiwan (R.O.C.) for awarding postdoctorship: NSC-102-2811-M-032-008 (August 2013-July 2014). Although, Dr. Yeh is co-author of this paper yet first author appreciates giving his time and arriving from Hsinchu to conduct TEM operation under the given directions.

\section{References}


[1] P. Kim, C. M. Lieber, Nanotube Nanotweezers, Science 286, (1999) 2148-2150.

[2] Z. Tang, N. A. Kotov, M. Giersig, Spontaneous Organization of Single CdTe Nanoparticles into Luminescent Nanowires, Science 297, (2002) 237-240.

[3] Z. Tang, Z. Zhang, Y. Wang, S. C. Glotzer, N. A. Kotov, Self-Assembly of CdTe Nanocrystals into Free-Floating Sheets, Science 314, (2006) 274-278.

[4] Y. Liu, X. Zhang, Metamaterials: a new frontier of science and technology, Chem. Soc. Rev. 40, (2011) 2494-2507.

[5] A. Kuzyk, et al. DNA-based self-assembly of chiral plasmonic nanostructures with tailored optical response, Nature 483, (2012) 311-314.

[6] J. Kim, Y. Lee, S. Sun, Structurally ordered FePt nanoparticles and their enhanced catalysis for oxygen reduction reaction, J. Am. Chem. Soc. 132, (2010) 4996-4997.

[7] K. Kusada, et al. Discovery of face-centered-cubic ruthenium nanoparticles: facile size-controlled synthesis using the chemical reduction method, J. Am. Chem. Soc. 135, (2013) 5493-5496.

[8] M. Brust, M. Walker, D. Bethell, D. J. Schiffrin, R. Whyman, Synthesis of Thiolderivatised Gold Nanoparticles in a Two-phase Liquid-Liquid System, J. Chem. Soc., Chem. Commun. (1994) 801-802.

[9] R. L. Whetten, et al. Nanocrystal Gold Molecules, Adv. Mater. 8, (1996) 428-433.

[10] L. O. Brown, J. E. Hutchison, Formation and Electron Diffraction Studies of Ordered 2-D and 3-D Superlattices of Amine-Stabilized Gold Nanocrystals, J. Phys. Chem. B 105, (2001) 8911-8916.

[11] M. Brust, C. J. Kiely, Some recent advances in nanostructure preparation from gold and silver particles: a short topical review, Colloids and Surfaces A: Physicochem. Eng. Aspects 202, (2002) 175-186.

[12] G. M. Whitesides, M. Boncheva, Beyond molecules: Self-assembly of mesoscopic and macroscopic components, Proc. Natl. Acad. Sci. USA 99, (2002) 4769-4774.

[13] J. Huang, F. Kim, A. R. Tao, S. Connor, P. Yang, Spontaneous formation of nanoparticle stripe patterns through dewetting, Nature Mater. 4, (2005) 896-900. 
[14] S. C. Glotzer, et al. Self-assembly of anisotropic tethered nanoparticle shape amphiphiles, Curr. Opin. Colloid Interface Sci. 10, (2005) 287-295.

[15] S. C. Glotzer, M. J. Solomon, Anisotropy of building blocks and their assembly into complex structures, Nature Mater. 6, (2007) 557-562.

[16] S. Link, M. A. El-Sayed, Shape and size dependence of radiative, nonradiative and photothermal properties of gold nanocrystals, Int. Rev. Phys. Chem. 19, (2000) 409- 453.

[17] D. Vanmaekelbergh, Self-assembly of colloidal nanocrystals as route to novel classes of nanostructured materials, Nano Today 6, (2011) 419-437.

[18] C. P. Shaw, D. G. Fernig, R. Lévy, Gold nanoparticles as advanced building blocks for nanoscale self-assembled systems, J. Mater. Chem. 21, (2011) 1218112187.

[19] Y. Negishi, et al. A Critical Size for Emergence of Nonbulk Electronic and Geometric Structures in Dodecanethiolate-Protected Au Clusters, J. Am. Chem. Soc. 137, (2015) 1206-1212.

[20] N. Liu, M. L. Tang, M. Hentschel, H. Giessen, A. P. Alivisatos, Nanoantennaenhanced gas sensing in a single tailored nanofocus, Nat. Mater. 10, (2011) 631637.

[21] N. Li, P. Zhao, D. Astruc, Anisotropic Gold Nanoparticles: Synthesis, Properties, Applications, and Toxicity, Angew. Chem. Int. Ed. 53, (2014) 1756-1789.

[22] M. L. Brongersma, N. J. Halas, P. Nordlander, Plasmon-induced hot carrier science and technology, Nature Nanotechnol. 10, (2015) 25-34.

[23] G. van Anders, D. Klotsa, N. K. Ahmed, M. Engel, S. C. Glotzer, Understanding shape entropy through local dense packing, Proc. Natl. Acad. Sci. U.S.A. 111, (2014) E4812-E4821.

[24] V. N. Manoharan, Colloidal matter: Packing, geometry, and entropy, Science 349, (2015).

[25] S. Atkinson, F. H. Stillinger, S. Torquato, Existence of isostatic, maximally random jammed monodisperse hard-disk packings, Proc. Natl. Acad. Sci. U.S.A. 111, (2015) 18436-18441. 
[26] W. -H. Chiang, C. Richmonds, R. M. Sankaran, Continuous-flow, atmosphericpressure microplasmas: a versatile source for metal nanoparticle synthesis in the gas or liquid phase, Plasma Sources Sci. Technol. 19, (2010) 034011-18.

[27] D. Mariotti, J. Patel, V. Švrček, P. Maguire, Plasma -Liquid Interactions at Atmospheric Pressure for Nanomaterials Synthesis and Surface Engineering, Plasma Process. Polym. 9, (2012) 1074-1085.

[28] J. Patel, L. Něemcová, P. Maguire, W. G. Graham, D. Mariotti, Synthesis of surfactant-free electrostatically stabilized gold nanoparticles by plasma -induced liquid Chemistry, Nanotechnology 24, (2013) 245604-14.

[29] X. Huang, Y. Li, X. Zhong, Effect of experimental conditions on size control of Au nanoparticles synthesized by atmospheric microplasma electrochemistry, Nanoscale Research Lett. 9, (2014) 572-578.

[30] N. Shirai, S. Uchida, F. Tochikubo, Synthesis of metal nanoparticles by dual plasma electrolysis using atmospheric dc glow discharge in contact with liquid, Jpn. J. Appl. Phys. 53, (2014) 046202-046207.

[31] C. Richmonds, et al. Electron-transfer reactions at the plasma-liquid interface, $J$. Am. Chem. Soc. 133, (2011) 17582-17585.

[32] M. Witzke, P. Rumbach, D. B. Go, R. M. Sankaran, Evidence for the electrolysis of water by atmospheric-pressure plasmas formed at the surface of aqueous solutions, J. Phys. D: Appl. Phys. 45, (2012) 442001-05.

[33] J. Hieda, N. Saito, O. Takai, Exotic shapes of gold nanoparticles synthesized using plasma in aqueous solution, J. Vac. Sci. Technol. A 26, (2008) 854-856.

[34] N, Saito, J. Hieda, O. Takai, Synthesis process of gold nanoparticles in solution plasma. Thin Solid Films 518, (2009) 912-917.

[35] M. A. Bratescu, S. -P. Cho, O. Takai, N. Saito, Size-Controlled Gold Nanoparticles Synthesized in Solution Plasma, J. Phys. Chem. C 115, (2011) 24569-24576.

[36] Y. K. Heo, M. A. Bratescu, D. Aburaya, N. A. Saito, phonon thermodynamics approach of gold nanofluids synthesized in solution Plasma, Appl. Phys. Lett. 104, (2014) 111902-05. 
[37] K, Furuya, Y. Hirowatari, T. Ishioka, A. Harata, Protective Agent-free Preparation of Gold Nanoplates and Nanorods in Aqueous $\mathrm{HAuCl}_{4}$ Solutions Using GasLiquid Interface Discharge, Chem. Lett. 36, (2007) 1088-1089.

[38] M. Ali, I $-\mathrm{N}$. Lin, The effect of the Electronic Structure, Phase Transition, and Localized Dynamics of Atoms in the formation of Tiny Particles of Gold. http://arxiv.org/abs/1604.07144.

[39] M. Ali, I -N. Lin, Development of gold particles at varying precursor concentration. http://arXiv.org/abs/1604.07508.

[40] M. Ali, I $-\mathrm{N}$. Lin, Tapping opportunity of tiny shaped particles and role of precursor in developing shaped particles. http://arxiv.org/abs/1605.02296.

[41] M. Ali, I -N. Lin, Controlling morphology-structure of particles at different pulse rate, polarity and effect of photons on structure. http://arxiv.org/abs/1605.04408.

[42] M. Ali, I -N. Lin, Formation of tiny particles and their extended shapes -Origin of physics and chemistry of materials. http://arxiv.org/abs/1605.09123.

[43] M. Ali, The study of tiny shaped particle dealing localized gravity at solution surface. http://arxiv.org/abs/1609.08047.

[44] M. Ali, Atoms of electronic transition deform or elongate but do not ionize while inert gas atoms split. http://arxiv.org/abs/1611.05392.

[45] M. Ali, Structure evolution in atoms of solid state dealing electron transitions. http://arxiv.org/abs/1611.01255.

[46] M. Ali, Revealing the Phenomena of Heat and Photon Energy on Dealing Matter at Atomic level. https://www.preprints.org/manuscript/201701.0028/v10.

[47] M. Ali, Nanoparticles-Photons: Effective or Defective Nanomedicine. J. Nanomed. Res. 5 (2017) 00139.

[48] M. Ali, M. Ürgen, Switching dynamics of morphology-structure in chemically deposited carbon films -A new insight, Carbon 122 (2017) 653-663.

[49] M. Ali, I -Nan. Lin, Phase transitions and critical phenomena of tiny grains carbon films synthesized in microwave-based vapor deposition system. http://arXiv.org/abs/1604.07152. 
[50] M. Ali, Why atoms of some elements are in gas state and some in solid state, but carbon on works either (2018).

https://www.preprints.org/manuscript/201803.0092/v2

\section{Authors' biography:}
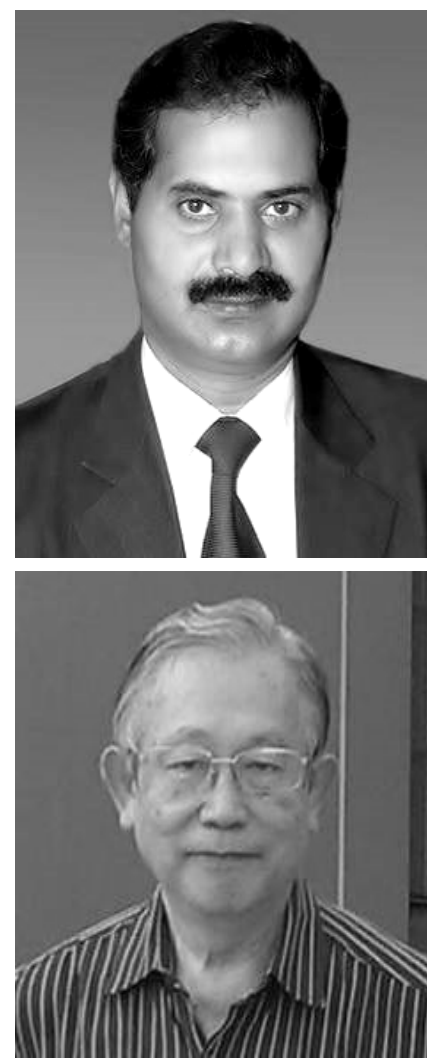

Mubarak Ali graduated from University of the Punjab with B.Sc. (Phys\& Maths) in 1996 and M.Sc. Materials Science with distinction at Bahauddin Zakariya University, Multan, Pakistan (1998); thesis work completed at Quaid-i-Azam University Islamabad. He gained Ph.D. in Mechanical Engineering from Universiti Teknologi Malaysia under the award of Malaysian Technical Cooperation Programme (MTCP;2004-07) and postdoc in advanced surface technologies at Istanbul Technical University under the foreign fellowship of The Scientific and Technological Research Council of Turkey (TÜBITAK; 2010). He completed another postdoc in the field of nanotechnology at Tamkang University Taipei (2013-2014) sponsored by National Science Council now M/o Science and Technology, Taiwan (R.O.C.). Presently, he is working as Assistant Professor on tenure track at COMSATS Institute of Information Technology, Islamabad campus, Pakistan (since May 2008) and prior to that worked as assistant director/deputy director at M/o Science \& Technology (Pakistan Council of Renewable Energy Technologies, Islamabad; 2000-2008). He was invited by Institute for Materials Research (IMR), Tohoku University, Japan to deliver scientific talk on growth of synthetic diamond without seeding treatment and synthesis of tantalum carbide. He gave several scientific talks in various countries. His core area of research includes materials science, physics \& nanotechnology. He was also offered the merit scholarship (for PhD study) by the Government of Pakistan but he couldn't avail. He is author of several articles published in various periodicals (https://scholar.google.com.pk/citations?hl=en\&user=UYjvhDwAAAAJ).

I-Nan Lin is a senior professor at Tamkang University, Taiwan. He received the Bachelor degree in physics from National Taiwan Normal University, Taiwan, M.S. from National TsingHua University, Taiwan, and the Ph.D. degree in Materials Science from U. C. Berkeley in 1979, U.S.A. He worked as senior researcher in Materials Science Centre in Tsing-Hua University for several years and now is faculty in Department of Physics, Tamkang University. Professor Lin has more than 200 referred journal publications and holds top position in his university in terms of research productivity. Professor I-Nan Lin supervised several PhD and Postdoc candidates around the world. He is involved in research on the development of high conductivity diamond films and on the transmission microscopy of materials. 